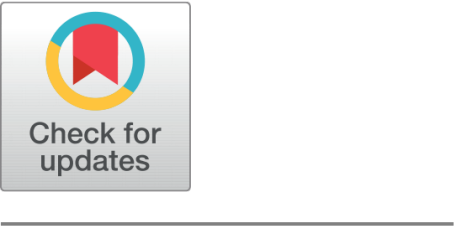

open acCess

Received: 28.07.2020

Accepted: 23.02.2021

Published: 09.03.2021

Citation: Yadav K, Saad SA (2021) Game theory based adaptive transmit power control algorithm for loT wireless sensor networks. Indian Journal of Science and Technology 14(7): 690-697. https://d oi.org/10.17485/IJST/v14i7.1258

* Corresponding author.

kusumasyadav0@gmail.com

Funding: None

Competing Interests: None

Copyright: @ 2021 Yadav \& Saad. This is an open access article distributed under the terms of the Creative Commons Attribution License, which permits unrestricted use, distribution, and reproduction in any medium, provided the original author and source are credited.

Published By Indian Society for Education and Environment (iSee)

ISSN

Print: 0974-6846

Electronic: 0974-5645

\section{Game theory based adaptive transmit power control algorithm for loT wireless sensor networks}

\author{
Kusum Yadav ${ }^{1}$, Sawsan A Saad ${ }^{1}$ \\ 1 College of Computer Science \& Engineering, University of Ha'il, Kingdom of Saudi Arabia
}

\section{Abstract}

Objectives: To design a transmission power control (TPC) algorithm for the Internet of Things (IOT) based Wireless Sensor Networks (WSN) such that the energy cost is reduced and the expected throughput is increased. Method: In this study, a Game theory based adaptive transmit power control (GT-ATPC) algorithm for IoT-WSN is proposed. In this algorithm, for each sender, the initial transmission power level (TPL) is determined from the difference of Received Signal Strength (RSS) measurements at the base station (BS). The TPL is adaptively adjusted by means of Game theory. For each sender, the expected throughput, energy cost and connectivity values are estimated from which a utility function is derived. Findings: By simulation results, it has been shown that GT-ATPC attains 37\% higher throughput with 57\% reduced energy cost and 30\% lesser overhead when compared with GTS-PM approach. Novelty: The TPL of a node is adjusted based on the estimated values of utility function such that the throughput and connectivity is high and energy cost is less.

Keywords: Internet of Things (IoT); wireless sensor network (WSN); transmission power; Energy cost; game theory; utility function

\section{Introduction}

The Internet of Things (IoT) is a system of several things which allows a unified movement among such physical stuff ${ }^{(1,2)}$. As a substitute to use extremely accessible broadcast power for every sensor node, it should regulate its broadcast power to the desired level for attaining a dependable communication network ${ }^{(3,4)}$. Energy administration scheme centered on IoT enables such necessities at comfort in a value-focused mode ${ }^{(5)}$. As the detecting expedients are battery-powered, a lowpower scheme is the chief requisite. ${ }^{(6)}$. Since IoT devices have low power and have lossy communication channels ${ }^{(7)}$, a Transmission power control (TPC) is one of the mechanisms used to enhance the lifetime of a sensor node. In TPC, the transmit power level (TPL) is adaptively adjusted based on the channel conditions ${ }^{(8)}$. However, existing works on TPC in IoT networks involve high computational and storage overhead ${ }^{(9)}$. Apart from channel condition metrics like Received Signal Strength Indicator (RSSI) and Packet Reception Rate (PRR), the expected throughput and connectivity metrics need to be considered during TPC. 
TPC can be achieved by means of effective topology control also. In ${ }^{(10)}$, a wireless topology control algorithm for WSN, based on an adaptive fuzzy neural control system has been proposed. In this algorithm, ANN is trained to obtain the parameters of the Fuzzy controller. The communication range of the nodes is constantly adjusted through feedback and circulation so as to control the transmission power of the node. Though it ensures low overhead, the feedback approach causes additional traffic in the network.

A power optimization framework for non-orthogonal multiple access (NOMA) enabled IoT devices have been designed in ${ }^{(11)}$. It combinedly addresses the issues of transmit power, interference and QoS. It minimizes the total transmitted power of IoT devices under the Successive Interference Cancellation (SIC) constraint. However, it does not consider the connectivity issues of IoT devices during movement.

An IoT-based management platform has been designed ${ }^{(12)}$ for dynamically adjusting the broadcasting network radiated power, based on the current propagation conditions. The power consumption of a broadcast network is optimized by monitoring fading throughout. However, it does not suitable for one-to-one based transmissions.

The concept of Game Theory (GT) has been applied in Wireless Personal Area networks, Body area networks and IoT networks for decision making and power control problems. The GT consists of Game, a set of players with a specific strategy. A Game can be any set of circumstances that has a result dependent on the actions of two of more decision-makers (players) The Player is a strategic decision-maker within the context of the game. A strategy is a complete plan of action a player will take for given circumstances that might arise within the game. A game is cooperative if the players are able to form binding commitments externally enforced or it is non-cooperative if players cannot form alliances or if all agreements need to be self-enforcing.

In Game Theoretic Solution for Power Management (GTS-PM) approach ${ }^{(13)}$, two games were used based on the evolutionary game model. The first game was applied for cluster head selection. The second game was used to assign equal tasks for the cluster heads. The key parameters used are the remaining energies, the hop levels, the degree of connectivity, the density of nodes and the selfishness level of nodes. But it does not adjust the power levels directly.

Game Theory has been applied for clustering ${ }^{(14)}$ in which Energy Efficient Clustering routing protocol (GEEC) has been developed. It adopts an evolutionary game theory mechanism to achieve energy exhaust equilibrium as well as lifetime extension at the same time. Game theory has been used to address the congestion problem ${ }^{(15)}$ using a parent-change procedure. In this procedure, the child nodes can change next hop neighbors towards the sink during congestion. Similar to Game theory, a distributed online learning solution ${ }^{(16)}$ has been designed for low-power IoT devices. In online learning, each device maximizes an objective function in terms of reliability and energy efficiency. However, it does not guarantee the expected throughput of successful communications.

In ${ }^{(17)}$, an evolutionary game theory-based slot allocation scheme has been proposed for the calculation of probability by solving Nash equilibrium point. This probability is used by mobile nodes to send data packets to a new cluster head. In ${ }^{(18)}$, Game theory based Energy Efficient Clustering routing protocol (GEEC) has been proposed. GEEC adopts evolutionary game theory mechanism to achieve energy exhaust equilibrium as well as lifetime extension at the same time.

In ${ }^{(19)}$ investigated unmanned aerial vehicle (UAV)-aided full-duplex wireless powered IoT networks, where a UAV equipped with a full-duplex hybrid access point (HAP) serves multiple sparsely distributed energy constrained IoT sensors. Under the proposed model, they formulated two problems: sum-throughput maximization (STM) and total-hover-time minimization (THTM). In Adaptive Transmission Power Control (ATPC) ${ }^{(20)}$ for WSN, each node builds a model for each of its neighbors, describing the correlation between transmission power and link quality. With this model, they employed a feedback-based transmission power control algorithm to dynamically maintain individual link quality over time. In ${ }^{(21)}$, a new power allocation technique has been designed for improving the energy and spectral efficiency of NOMA-enabled IoT devices. By considering the transmit power, QoS and successive interference cancellation (SIC) constraints, they used the sequential quadratic programming (SQP) technique to solve the non-convex problem.

Hence, there is a lack of works on Game theory based TPC control in IoT networks. The main focus of this work is to design a TPC algorithm for IoT networks such that:

- Determine the transmit power adaptively using Game theory

- Reduce the energy cost involved in overhead

- Increase the expected throughput

- Ensure required connectivity irrespective of the location

This study proposes Game theory based adaptive transmit power control (GT-ATPC) algorithm for IoT-WSN. 


\section{Game theory based adaptive transmit power control (GT-ATPC algorithm}

\subsection{Overview}

In this article, game theory based adaptive TPC algorithm for IoT WSN is proposed. For each neighbor, the initial TPL is determined from the difference of RSS measurements at the base station (BS). The TPL is adaptively adjusted by means of Game theory. For each sender, the expected throughput, energy cost and connectivity values are estimated from which a utility function is derived. Then TPL of a node is adjusted based on the estimated values of utility function such that the throughput and connectivity are high and energy cost is less. Figure 1 shows the block diagram of GT-ATPC technique.
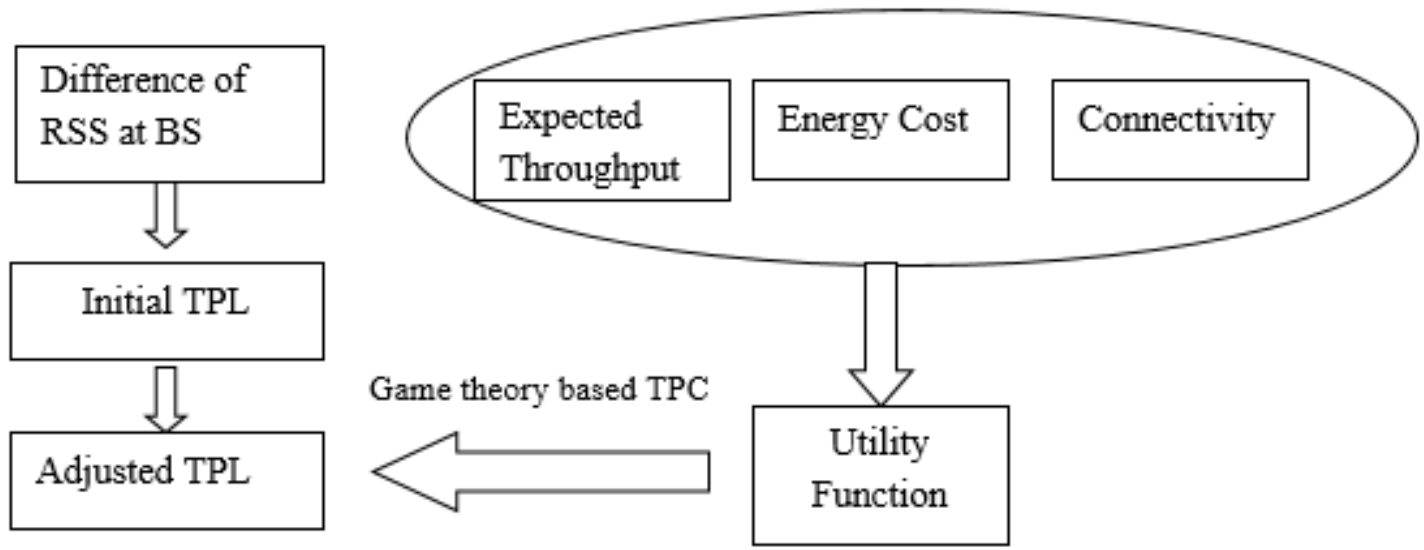

\section{Maximize Throughput, Reduce Energy Cost}

Fig 1. Block diagram of GT-ATPC

\subsection{Estimation of energy cost}

Every node functions on a partial group of energy. As the entire nodes are supposed to the intellect, practice, combined, direct, accept and forward the data, they will ingest dynamisms with the track of period.

Let $\mathrm{E}_{i}$ denotes the energy consumed by node $\mathrm{N}_{i}$. Then energy cost (ECost) of $\mathrm{N}_{i}$ is represented by

$$
E \text { Cost }_{i}=\operatorname{Cost}_{i}(\text { Sense })+\text { Cost }_{i}(\text { Process })+\operatorname{Cost}_{i}(\text { agg })+\operatorname{Cost}_{i}(P T x)
$$

Where $\operatorname{Cost}_{i}(P T x)$ can be formed for aggregators AGG as:

$$
\operatorname{Cost}_{A G}(P T x)=\text { Cost }_{t x(A G, B S)}+\text { Cost }_{r x(\operatorname{agg})}
$$

here, $\operatorname{Cost}_{t x(A G, B S)}$ is the Ecost for packet transmission from AG to BS. Cost $t_{r x(a g g)}$ is the Ecost of packet reception from the sensor nodes and aggregating.

The term $\operatorname{Cost}_{t x(A G, B S)}$ is redefined as:

$$
\operatorname{Cost}_{t x(A G, B S)}=d_{A G, B S}^{2} E(a m p)+E(\text { elec })
$$

Where, $d_{A G, B S}^{2}$ is the distance between AG and BS, E(amp) and $E($ elec $)$ are the amplifier and the circuitry dissipations.

The cost of receiving and aggregating packets is given by

$$
\operatorname{Cost}_{r x(\mathrm{agg})}=\sum_{i=1}^{k} d_{i}^{2} \cdot E(\text { elec })+k \cdot E(\mathrm{agg})+E(\text { lis })
$$

In Eq.(4), we have a set of connected node $\mathrm{N}_{i}, \mathrm{i}=1,2 \ldots \mathrm{k}, \mathrm{d}_{i}$ is the distance between $\mathrm{AG}$ and $\mathrm{N}_{i} . \mathrm{E}(\mathrm{lis})$ is the energy consumed in antenna listening. 


\subsection{Estimation of connectivity}

As the nodes are arbitrarily disseminated in the system, their connectivity degree $\left(\mathrm{D}_{\text {con }}\right)$ with other nodes may be dissimilar. $\mathrm{D}_{\text {conn }}$ with the $\mathrm{BS}$ is mainly estimated by the number of hops.

Hence, $\mathrm{D}_{c o n}$ can be estimated by considering its possible downstream $\left(\right.$ Down $\left._{s t r}\right)$ and upstream $\left(\mathrm{Up}_{s t r}\right)$ nodes as

$$
D_{\text {con } n}=\frac{\text { No Do } w n_{s t r}+1}{\text { NoUp } p_{s t r}+1}
$$

Where NoDown str $_{\text {and NoUp }}$ str denote the number of downstream and upstream nodes of $\mathrm{N}_{i}$.

\subsection{Estimation of expected throughput}

The RSS at each node from BS is estimated as:

$$
r S S_{d}=p_{1}-10 \alpha \log _{10} d-\sum_{k} n_{k} w_{k}
$$

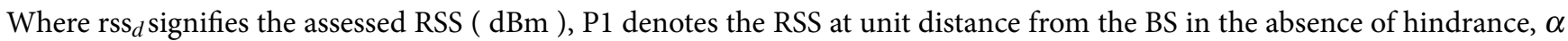
denotes the path loss exponent, $\mathrm{n}_{k}$ denotes the number of type $\mathrm{k}$ hindrances along the track from $\mathrm{BS}$ to $\mathrm{N}_{i}$ and $\mathrm{W}_{k}$ denotes the signal attenuation factor for $\mathrm{k}^{\text {th }}$ obstacle.

Then, the estimated throughput This computed by:

$$
T h=\frac{1}{1+e^{-\left(\frac{120+r S S_{d}-b}{c}\right)}}
$$

Where $\mathrm{a}, \mathrm{b}$ and $\mathrm{c}$ are weighted constants.

\subsection{Initial transmission power for AP}

Let rss $_{m}$ denotes the RSS measured at the BS, when maximum TPL is used by the nodes.

The difference between $\mathrm{rss}_{m}$ and $\mathrm{rss}_{d}$ is given by

$$
\Delta r S S=r S S m-r S S d
$$

Then the initial transmission power, $\mathrm{TP}_{0}$ is assessed as

$$
T P_{0}=T x \max -\Delta r s s
$$

Where $\mathrm{Tx}_{\max }$ denotes the maximum TPL of the AP. Here, $\mathrm{TP}(0)$ need to be in the possible series:

\subsection{Game theory based TPC}

\subsubsection{Game theory}

Game theory is used in decision-making applications and power control problems.

A Game $\mathrm{Z}$ can be defined as

$$
Z=\left(N, S,\left\{U F_{i}\right\}\right)
$$

Where $\mathrm{N}$ denotes a set of players

$\mathrm{S}$ signifies deed space designed as a Cartesian product. i.e. $\mathrm{S}=\mathrm{S}_{1} \times \mathrm{S}_{2} \times \mathrm{S}_{3} \times \mathrm{S}_{4} \times \ldots \mathrm{S}_{\mathrm{n}}$

$\mathrm{UF}_{i}$ signifies the utility functions provided by

$$
U F_{i}=\left\{U F_{1}, U F_{2}, \ldots U F_{n}\right\}
$$

The consequences are nominated by a specific participant with $S_{i}$ as $\mathrm{UF}_{i}$ and the specific activities designated by other performers is $\mathrm{S}_{-i}$. 


\subsubsection{Utility function for TPC}

In this model, a utility function in terms of energy cost, connectivity, and throughput values is formed.

$$
U F(i)=\left(w 1 . D_{\text {coni }}+w 2 \cdot T h_{i}\right) / \operatorname{Cost} E_{i}
$$

At this time, Game theory model is used in which every participant aptly chooses the best $\mathrm{TPL}_{i}$ to maximize their utility function.

\subsubsection{Game theory based Adaptive TPC}

After setting the initial transmit power $\mathrm{TP}_{0}$ using Eq. (9), the transmit power of each node is adaptively adjusted by applying the Game theory model

$$
\text { (ie) } \begin{aligned}
T P & =T P_{0}, \text { if } U F(i) \text { is maximum } \\
& =T P_{\max }, \text { if } U F(i) \text { is min imum } \\
& =T P_{o}+\mu, U F(i) \text { otherwise }
\end{aligned}
$$

Where $T P_{\max }$ is the maximum TPL of node $N_{i}$.

$\mu$ is the small increment factor for $\mathrm{TP}_{0}$.

\section{Results and Discussion}

\subsection{Experimental settings}

The GT-ATPC algorithm is implemented in NS2 and compared with Game Theoretic Solution for Power Management (GTSPM) approach ${ }^{(13)}$. In simulation experiments, 50 to 150 nodes are deployed in an area of $50 \mathrm{~m} \mathrm{X} \mathrm{50m.} \mathrm{The} \mathrm{MAC} \mathrm{protocol} \mathrm{used}$ is IEEE 802.15.4. Initial energy assigned to all nodes is 20 joules, the transmission and reception powers are fixed as 0.8 and 0.5 watts, respectively.

\subsection{Results}

In simulation experiments, the number of nodes is varied as 50,75, 100, 125 and 150.

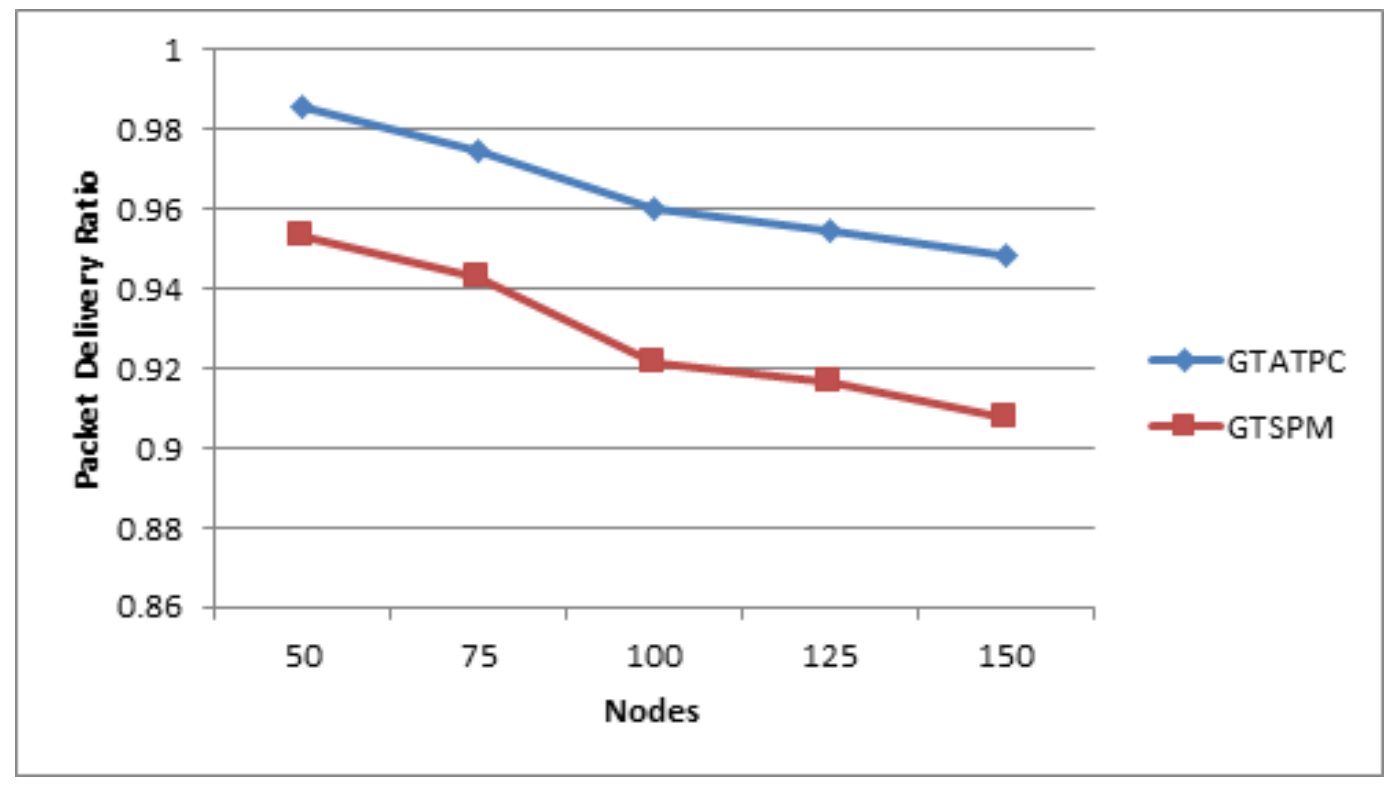

Fig 2. Packet delivery ratio of nodes

Figure 2 shows the packet delivery ratio of both techniques. Since GTATPC maintains optimum power level to ensure connectivity, it attains a higher delivery ratio than GTSPM which concentrates mainly on energy reduction factors only. Hence, it can be seen that the packet delivery ratio of GTATPC is $4 \%$ higher than GTSPM. 


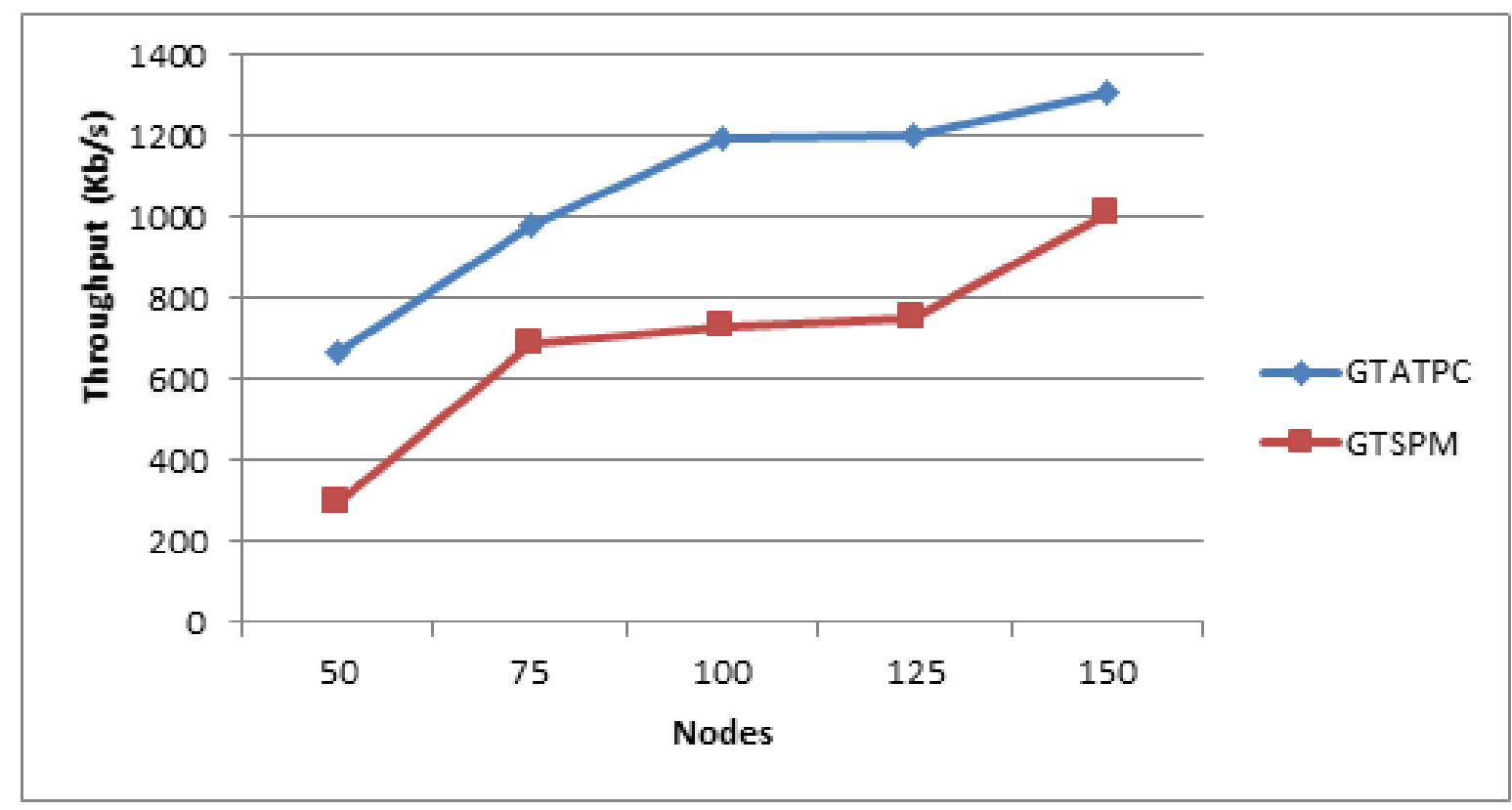

Fig 3. Expected throughput for nodes

Figure 3 shows the expected throughput measured for both techniques. Since GTATPC aims to increase the throughput through the utility function, it attains higher throughput than GTSPM which concentrates mainly on energy reduction factors only. It can be observed that the throughput of GTATPC is 37\% higher than GTSPM.

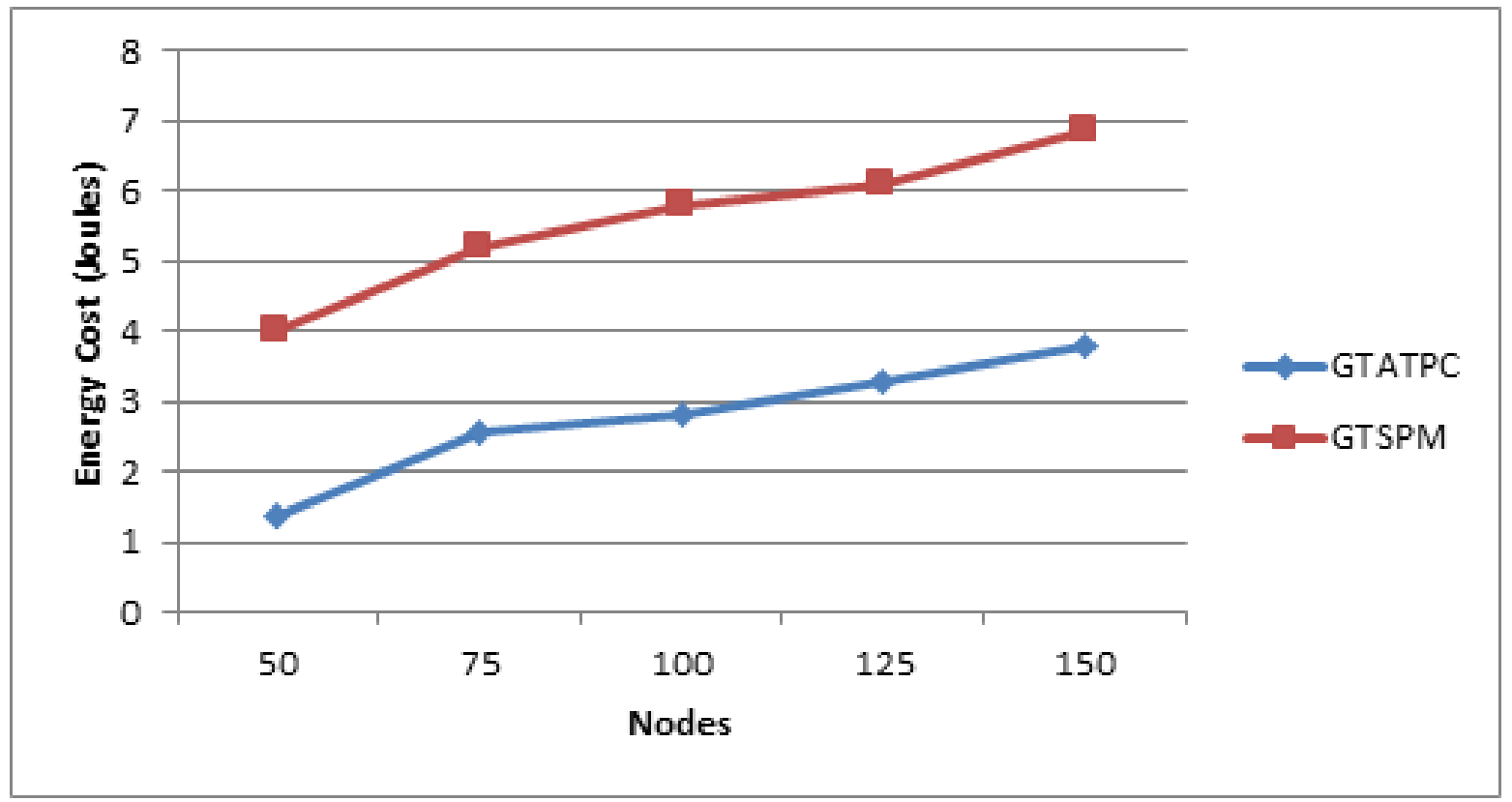

Fig 4. Energy cost for nodes

Figure 4 shows the energy cost measured by both techniques. Though GTSPM reduces the energy factor during the $\mathrm{CH}$ selection and task scheduling, it did not ensure minimum power level during transmission. Hence, it can be seen that the energy cost of GTATPC is $51 \%$ lesser than GTSPM. 


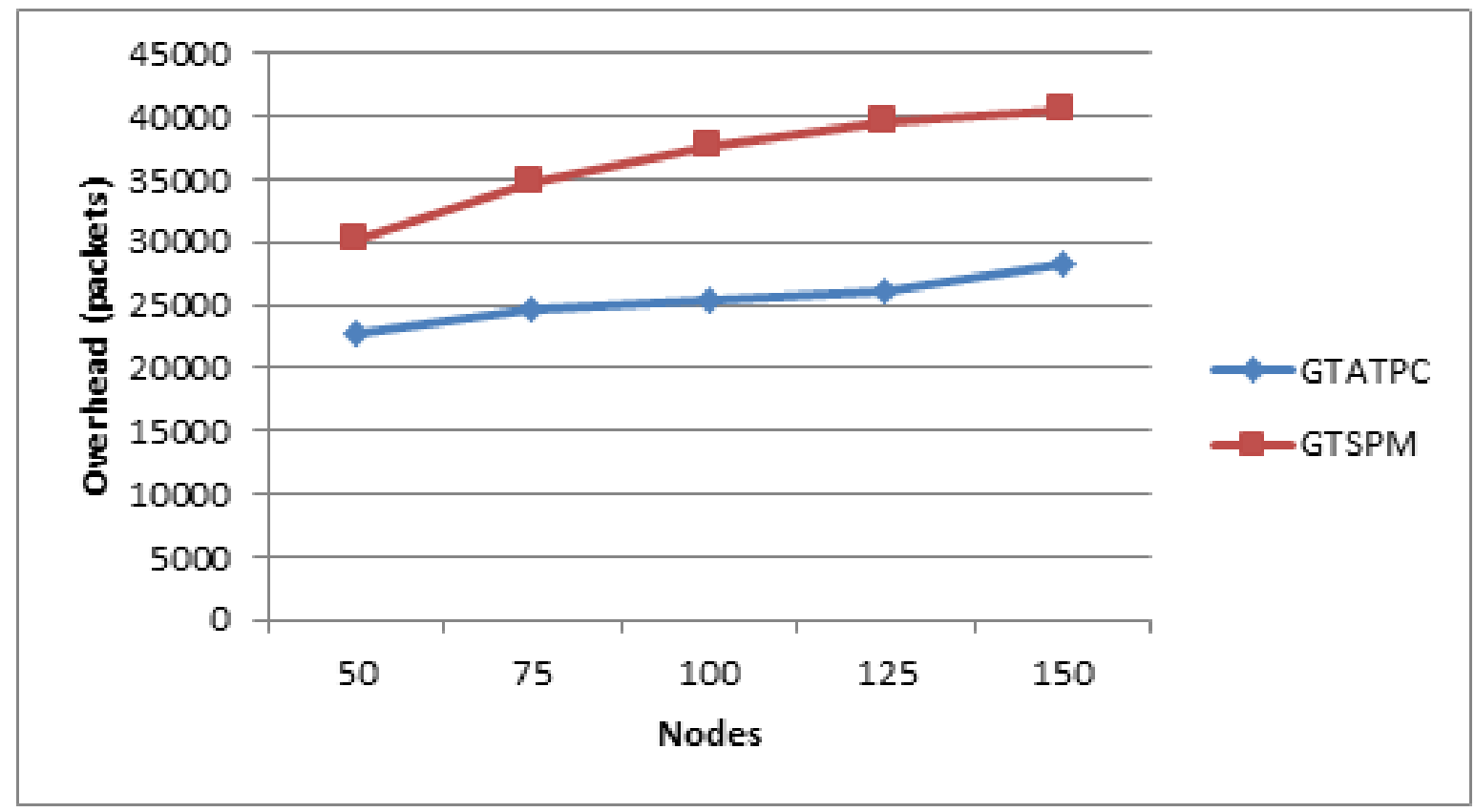

Fig 5. Energy cost for nodes

Figure 5 shows the overhead involved in both the techniques since GTAPC assures connectivity at the cost of reduced power level, the overhead involved in retransmissions and waiting are reduced. Hence, it attains 30\% lesser overhead compared to GTSPM.

\section{Conclusion}

This study proposes Game theory based adaptive transmit power control (GT-ATPC) algorithm for IoT-WSN. In this algorithm, for each sender, the initial TPL is determined from the difference of RSS measurements at the base station (BS). The TPL is adaptively adjusted by means of Game theory. For each sender, the expected throughput, energy cost and connectivity values are estimated from which a utility function is derived. Then TPL of a node is adjusted based on the estimated values of utility function such that the throughput and connectivity are high and energy cost is less. The proposed GT-ATPC algorithm is implemented in NS2 and compared with GTS-PM approach. The novelty of this work is that it adjusts the transmit power adaptively such that the energy cost involved in the device operation is minimized up to $57 \%$ and the expected throughput is maximized up to $37 \%$ when compared with the GTS-PM approach.

\section{References}

1) Razaque A, Oddo P, Amsaad HF, Sangavikar M, Manchikatla S, Niraj. Power Reduction for Smart Homes in an Internet of Things Framework. IEEE. 2016. Available from: https://doi.org/10.1109/EIT.2016.7535225,2016.

2) Shokrnezhad M, Khorsandi S. Joint Power Control and Channel Assignment in Uplink IoT Networks: A Non-Cooperative Game and Auction Based Approach. Computer Communications. 2018. Available from: https://doi.org/10.1016/j.comcom.2018.01.002.

3) Lynggaard P, Blaszczyk T. An Energy-Efficient Link with Adaptive Transmit Power Control for Long Range Networks. In: and others, editor. Global Wireless Summit. Proceedings. 2016. Available from: https://orbit.dtu.dk/en/publications/.

4) Sindhuja P, Balamurugan MS. Smart Power Monitoring and Control System through Internet of things using Cloud Data Storage. Indian Journal of Science and Technology. 2015;8(19). Available from: https://dx.doi.org/10.17485/ijst/2015/v8i19/76698.

5) Joy A, Manivannan D. Smart Energy Management and Scheduling using Internet of Things. Indian Journal of Science and Technology. 2016;9(48). Available from: https://dx.doi.org/10.17485/ijst/2016/v9i48/108001.

6) Kaur K, Kaur K. A Study of Power Management Techniques for Internet of Things (IoT). IEEE, International Conference on Electrical, Electronics, and Optimization Techniques (ICEEOT). 2016. Available from: https://doi.org/10.1109/ICEEOT.2016.7754992.

7) Khan ZA. Using energy-efficient trust management to protect IoT networks for smart cities. Sustainable Cities and Society. 2018;40:1-15. Available from: https://dx.doi.org/10.1016/j.scs.2018.03.026.

8) Alrajeh NA, Khan S, Campbell CEA, Shams B. Multi-Channel Framework for Body Area Network in Health Monitoring. Applied Mathematics \& Information Sciences. 2013;7(5):1743-1747. Available from: https://dx.doi.org/10.12785/amis/070511. 
9) Chawda P, Prasad A. Power Management Factors and Techniques for IoT Design Devices. In: 19th International Symposium on Quality Electronic Design. 2018.

10) Li F. A novel wireless topology control algorithm with energy balance and low-overhead based on adaptive fuzzy neural network". Journal of Ambient Intelligence and Humanized Computing. 2019. Available from: https://doi.org/10.1007/s12652-019-01461-5.

11) Khan WU, Jameel F, Jamshed MA, Pervaiz H, Khan S, Liu J. Efficient power allocation for NOMA-enabled IoT networks in 6G era. Physical Communication. 2020;39. Available from: https://dx.doi.org/10.1016/j.phycom.2020.101043.

12) Alonso RM, Plets D, Pupo EF, Deruyck M, Martens L, Nieto GG, et al. IoT-Based Management Platform for Real-Time Spectrum and Energy Optimization of Broadcasting Networks. Wireless Communications and Mobile Computing. 2018;2018:1-14. Available from: https://dx.doi.org/10.1155/2018/7287641.

13) Sohail M, Khan S, Ahmad R, Singh D, Lloret J. Game Theoretic Solution for Power Management in IoT-Based Wireless Sensor Networks. Sensors. 2019;19(18):3835-3835. Available from: https://dx.doi.org/10.3390/s19183835.

14) Lin D, Wang Q. A game theory based energy efficient clustering routing protocol for WSNs. In: and others, editor. Wireless Networks. Springer. 2017. Available from: https://doi.org/10.1007/s11276-016-1206-2.

15) Ma C, Sheu JP, Hsu CX. A Game Theory Based Congestion Control Protocol for Wireless Personal Area Networks. Journal of Sensors. 2016;2016:1-13. Available from: https://dx.doi.org/10.1155/2016/6168535.

16) Azari A, Cavdar C. Self-organized Low-power IoT Networks: A Distributed Learning Approach. IEEE Globecom. 2018. Available from: https: //doi.org/10.1109/GLOCOM.2018.8647894.

17) Maheshwari P, Sharma AK, Verma K. Game theoretic application for energy efficient mobility handling in wireless sensor network. Transactions on Emerging Telecommunications Technologies. 2020;31(9). Available from: https://dx.doi.org/10.1002/ett.4052.

18) Lin D, Wang Q. A game theory based energy-efficient clustering routing protocol for WSNs. Wireless Networks. 2016;23(4):1101-1111. Available from: https://doi.org/10.1007/s11276-016-1206-2,2016.

19) Kang K, Ye R, Pan Z, Liu J, Shimamoto S. Full-Duplex Wireless Powered IoT Networks. IEEE Access. 2018;6:53546-53556. Available from: https: //dx.doi.org/10.1109/access.2018.2872024.

20) Lin S, Miao F, Zhang J, Gang Z, gu L, Stankovic J, et al. ATPC: Adaptive Transmission Power Control for Wireless Sensor Networks. ACM Transactions on Sensor Networks. 2015. Available from: https://doi.org/10.1145/2746342.

21) Khan HS, Liu J. Efficient power allocation for NOMA-enabled IoT networks in 6G era. Physical Communication. 2020. Available from: https: //doi.org/10.1016/j.phycom.2020.101043,2020. 